\title{
Cidades Inteligentes: $O$ valor público da inovação disruptiva para geogovernança ${ }^{1}$
}

\section{Smart Cities: The public value of disruptive innovation for geogovernance}

\author{
Oliveira Queiroz, Andréa'; Souza Fernandes, Ricardo Augusto2; \\ ' Universidade Federal de São Carlos, Brasil e andolq@hotmail.com \\ 2 Universidade Federal de São Carlos e ricardo.asf@gmail.com
}

\begin{abstract}
RESUMO
Na sociedade da era digital, o uso crescente das tecnologias da informação e comunicação (TIC) impulsiona cada vez mais as inovações disruptivas como parte da solução às questões complexas e contraditórias que envolvem o dinâmica do desenvolvimento das cidades. Tais questões requerem uma gestão urbana eficiente. Assim, a governança é algo fundamental e, portanto, é preeminente tornar as cidades inteligentes - qualificada com as TICs para enfrentar, inclusive de forma criativa, os desafios urbanos. Desta forma, o presente estudo tem o objetivo de analisar o valor público da aplicação de inovações disruptivas, como exemplo a aplicação de crowdsourcing em conjunto aos sistemas de informação geográficas (SIG), de forma a efetivar a geogovernança em cidades inteligentes. Trata-se de parte do referencial teórico da dissertação de mestrado em Engenharia Urbana, desenvolvido por meio de revisão bibliográfica, auxiliada pela aplicação de técnicas bibliométricas para escolha das referências bibliográficas. Dos resultados obtidos, verifica-se que aplicações de crowdsourcing e outros tipos de participação cidadã colaboram para a melhor gestão urbana, qualificando com valor público as estratégias e ações de governança que uma cidade inteligente requer.
\end{abstract}

Palavras-chave: Crowdsourcing, Geotecnologia, Governança Urbana.

\begin{abstract}
At the digital era society, the growing use of information technology and communication (ITC) draws the disruptive innovations towards becoming part of complex and contradictory solutions regarding urban dynamic development. Such matters demand an efficient urban management, and governance is of importance to evolve to smart cities - to cope creatively with urban challenges using ITCs. This essay focus to assess the value of the use of disruptive innovations to the public, as an example of crowdsourcing combined to the geographical information systems (GIS), in order to enforce geogovernance in smart cities. The text refers to part of the theoretical essay of the Master in Urban Engineering, developed using bibliography

${ }^{1}$ QUEIROZ, Andréa O.; FERNANDES, Ricardo A. S. Cidades Inteligentes: O valor público da inovação disruptiva para geogovernança. In: II SIMPÓSIO NACIONAL DE GESTÃO E ENGENHARIA URBANA: SINGEURB, 2019, São Paulo. Anais... Porto Alegre: ANTAC, 2019.
\end{abstract}


and biometrics techniques. As result, it is possible to confirm that crowdsourcing and other methods of open participation work efficiently with urban management, applying strategic governance actions that an intelligent city requires.

Keywords: Crowdsourcing, Geotechnology, Urban Governance.

\section{INTRODUÇÃO}

A difusão das tecnologias da informação e comunicação (TIC) marca o novo paradigma da atual sociedade, impulsionado pela expansão da linguagem digital que gera, armazena, recupera, processa e transmite informação, criando ciclos cumulativos entre inovação e uso (CASTELLS, 2016). Assim, as cidades do século 21 não se conectam apenas pelas malhas rodoviárias, mas pelas redes de dados (TOWNSEND, 2013).

As TIC impactam nas transformações urbanas, sendo fundamental repensar as formas da gestão de cidades, quanto aos sistemas de transporte, recursos hídricos, resíduos, energia e ambiente natural, lidando de forma sustentável com as questões do crescimento e demanda desses recursos(OJO; DZHUSUPOVA; CURRY, 2016). Neste cenário, em 1990, o movimento conhecido por "crescimento inteligente" - defensor da criação e implantação de políticas urbanas inovadoras, marca o surgimento do conceito de cidade inteligente (HARRISON; DONNELLY, 2011).

A respeito de cidades inteligentes, um consenso conceitual ainda não está firmado(NAM; PARDO, 2011; CHOURABI et al., 2012; MEIJER; GIL-GARCIA; BOLÍVAR, 2016; PRZEYBILOVICZ; CUNHA; TOMOR, 2017), mas em síntese as frentes abrangidas com foco tecnológico, recursos humanos e governança são as recorrentes (NAM; PARDO, 201 1; MEIJER; BOLÍVAR, 2016). Neste viés, Ojo, Dzhusupova e Curry (2015), elencam aspectos que a envolve, que são: (1) governança participativa; (2) desenvolvimento de capital humano, de infraestrutura de TIC e da cidadania ativa; (3) inovação tecnológica, organizacional e política.

Disto, entende-se que governança inteligente é uma questão fundamental (GIL-GARCIA; PARDO; NAM, 2015; VIALE PEREIRA et al., 2017). Contudo, não se limita à questão tecnológica, mas ao processo complexo de mudança institucional (MEIJER; BOLÍVAR, 2016), sendo indispensável a ativação espacial, de modo a permitir a organização e gestão dos processos espaciais (ROCHE, 2014), viabilizando a geogovernança - que é governança sobre, para e pelos territórios (VINCENT et al., 2012), na qual a opinião dos habitantes é considerada no planejamento sustentável (VINCENT, 2008).

Para tanto, Meijer, Gil-Garcia e Bolívar (2016), defendem o desenvolvimento de estratégias para cidades inteligentes vinculado ao entendimento das condições contextuais, modelos de governança e valor público. Assim, chega-se ao conceito de valor público, pioneiramente definido por Mark Moore, nos anos 90 (THOMPSON et al., 2014; MONTEVERDE et al., 2017), como uma estrutura orientativa à tomada de decisão dos gestores públicos, em atendimento às necessidades do público em relação aos benefícios substantivos, bem como ao valor intrínseco de um governo melhor (HARRISON et al., 2011).

Assim, pergunta-se: o uso de inovações disruptivas, a exemplo, o uso de crowdsourcing ${ }^{2}$ em conjunto aos sistemas de informação geográficas (SIG), como instrumento de geogovernança efetivam com valor público as cidades inteligentes?

\section{PROCEDIMENTOS METODOLÓGICOS}

A governança urbana em cidades inteligentes é o objeto de estudo e o objetivo principal é a analisar do uso das inovações disruptivas como contribuição à criação de valor público.

Fundamenta-se em marcos teóricos, elaborados a partir da revisão bibliográfica, auxiliada

\footnotetext{
${ }^{2}$ Crowdsourcing - pode ser definido como uma fonte de informações vindas de uma multidão. Trata-se da união de pessoas para que juntas criarem conteúdo ou desenvolverem novos produtos, ou ainda, encontrarem soluções. Geralmente, consiste num trabalho colaborativo e voluntário e, portanto, pode ser entendida como Colaboração Coletiva ou como Participação Colaborativa.
} 
pela aplicação de técnicas bibliométricas na escolha das referências. Obtendo como produto a síntese de alguns conceitos, tais como: crowdsourcing, governança inteligente, geogovernança e valor público.

\section{GEOGOVERNANÇA E O VALOR PÚBLICO DAS INOVAÇÕES DISRUPTIVAS}

Na perspectiva que "uma cidade inteligente precisa de um governo mais inteligente e governança mais inteligente" (GIL-GARCIA; PARDO; NAM, 2015b, p.6) associada tanto às estratégias inovadoras que permitem estruturas governamentais mais ágeis e resilientes, quanto às infraestruturas de governança (PRZEYBILOVICZ; CUNHA; TOMOR, 2017). Portanto, a governança inteligente é repensar e reinventar o governo a partir de um modelo mais participativo, transparente, democrático e responsivo(TOWNSEND, 2013).

A evolução dos governos abertos são a nova tendência que rompe a noção tradicional de gestão pública (GÓMEZ; CRIADO; GIL-GARCIA, 2017), fazendo que os governos locais estabeleçam como prioridade o governo eletrônico(CALDERON; LOPEZ; MARIN, 2018), que pelo uso das TIC viabilizam melhorias na participação política, na implementação das políticas públicas, ou no fornecimento das serviços do setor público (BOLÍVAR, 2016).

Assim, governo aberto e eletrônico entrelaçam-se ao conceito de governo inteligente, visto combinar o conjunto de processos de negócios e recursos tecnológicos para avançar na melhoria da entrega de informações e serviços públicos para a sociedade (VIALE PEREIRA et al., 2017a).

Sendo a governança eletronicamente mediada uma importante forma de incorporar diversos atores às decisões urbanas e, conforme inúmeros estudos apontam, tornar-se cada vez mais digitalizada permite lidar a inovação urbana com maior amplitude. Nesta categoria destacam-se 3 elementos: (1) governança eletrônica; (2) engajamento de stakeholders, cidadãos e comunidades; e (3) redes, parcerias e colaboração (GIL-GARCIA; PARDO; NAM, 2015a).

Além desta ativação digital, mostra-se imprescindível a ativação espacial. Portanto, a interrelação de governança e o uso de Sistemas de Informações Geográficas (SIG) é necessária. Sendo o SIG é uma ferramenta de aprimoramento à governança, e mesmo que sejam instrumentos imperativos, estes não dispensam incorporar a participação popular (MCCALL, 2003) para promover a efetiva Geogovernança.

\section{APRESENTAÇÃO E ANÁLISE DOS RESULTADOS}

Compreende-se a colaboração inovadora como ferramenta à boa governança em uma cidade inteligente, que produz ampla gama de valores públicos (MEIJER; BOLíVAR, 2016). Sendo a criação de um ambiente de colaboração as principais diferenças entre o governo eletrônico e os conceitos de governança inteligente (VIALE PEREIRA et al., 2017b).

Da Figura 1, percebe-se que a governança urbana administra tipos básicos de impactos de valor e, quando conectados aos princípios de boa governança geram valor público. 0 mesmo acontece na governança inteligente e, portanto, na geogovernança, quando promovem os princípios de governo aberto somando a ativação espacial, por meio do SIG e crowdsourcing. 
Figura 1 - Contexto de governança em cidades inteligentes e valor público

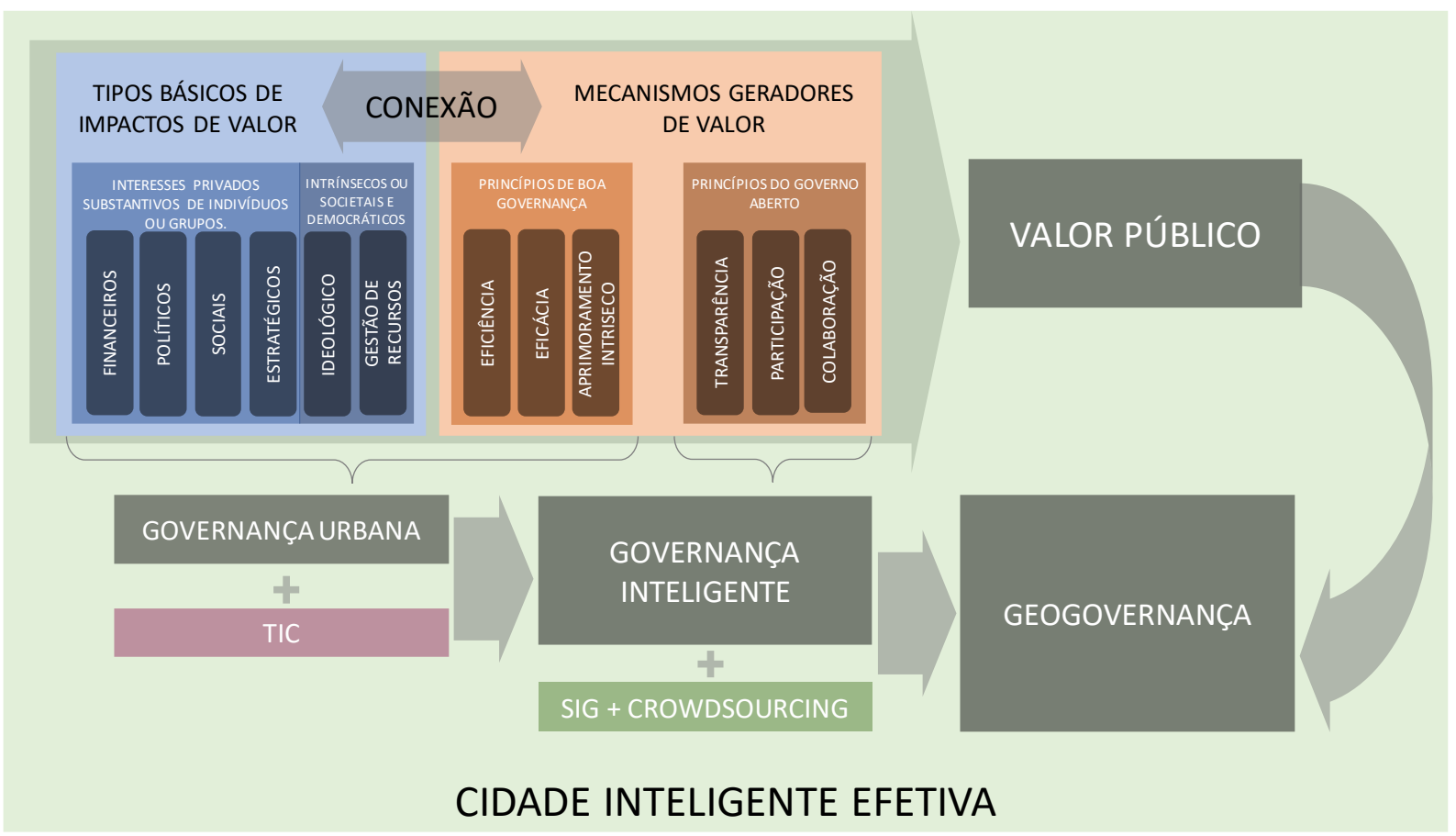

Fonte: Autores (2019)

Tais tipos de dados e funções de modelagem são fundamentais para construir a governança eletrônica e conectar informações individuais com a participação cooperativa (BATTY et al., 2012), possibilitando usar variados processos de crowdsourcing, gerando conscientização pública sobre questões sociais (HALDER, 2014), estabelecendo as colaborações como resposta legal às regras ou às tomadas de decisão pelo governo (RØISELAND, 2011).

Observa-se, como um dos benefícios associados ao crowdsourcing, o fornecimento de informações de alta qualidade e eficazes para projetos científicos com ganhos sociais e ambientais conquistados pela sensibilização e coleção de dados em escala e medida, improváveis de conseguir em projetos regulares (ATTARD; HAKLAY; CAPINERI, 2016).

Disto, finaliza-se de maneira sinóptica com o quadro 1, que traz os conceitos relacionados aos objetivos do estudo, resultado da revisão bibliográfica, que são: cidade inteligente, crowdsourcing, geogovernança, governança inteligente e, por fim, valor público.

Quadro 1 - Síntese de Conceitos

\begin{tabular}{l|l}
\hline termo & definição \\
\hline inteligente & O rótulo de "Cidade Inteligente" está relacionado à capacidade de pessoas \\
inteligentes gerarem soluções inteligentes para os problemas urbanos (ALBINO; \\
BERARDI; DANGELICO, 2015). Melhor dito, como as que envolvem a criação de \\
novas relações entre tecnologia e sociedade (MEIJER; GIL-GARCIA; BOLIVAR, \\
2016), concebidas pelas capacidades de aprendizado, desenvolvimento \\
tecnológico e inovação aplicadas aos processos de gestão da dinâmica urbana \\
e sustentadas nas infraestruturas digitais (HERNÁNDEZ-MUNOZ et al., 2011; \\
KOMNINOS; PALLOT; SCHAFFERS, 2011), ou ainda, como define a ISO-37122: \\
"[...] é aquela que aumenta o ritmo no qual ela fornece resultados \\
de sustentabilidade social, econômica e ambiental. [...] \\
respondem a desafios como mudança climática, rápido \\
crescimento populacional e instabilidade política e econômica, \\
melhorando fundamentalmente a forma como envolvem a \\
sociedade, aplicam métodos de liderança colaborativos,
\end{tabular}




\begin{tabular}{|c|c|}
\hline termo & definição \\
\hline & $\begin{array}{l}\text { trabalham entre disciplinas e sistemas municipais e usam } \\
\text { informações de dados e tecnologias modernas para oferecer } \\
\text { melhores serviços e qualidade de vida para aqueles na cidade } \\
\text { (moradores, empresas, visitantes), agora e no futuro previsível, sem } \\
\text { desvantagem injusta de outros ou degradação do ambiente } \\
\text { natural". }\end{array}$ \\
\hline Crowdsourcing & $\begin{array}{l}\text { Refere-se a novas formas de participação à coleta e à geração de informações } \\
\text { por grupos de usuários/contribuidores (HEIPKE, 2010; BASIRI, AMIRIAN, MOONEY, } \\
2016 \text { ), possibilitado pelo poder da rede (BATTY et al., 2012), envolve a coleta de } \\
\text { dados geoespaciais (BROVELLI; MINGHINI; ZAMBONI, 2016). Relaciona-se às } \\
\text { informações geográficas voluntárias, inclusive, pela possibilidade de solucionar } \\
\text { problemas (GOODCHILD; GLENNON, 2010), ou seja, são ações de cidadãos que } \\
\text { fornecem informações úteis à gestão das cidades, que auxilia nas tomadas de } \\
\text { decisão (SANGIAMBUT; SIEBER, 2016), pois dados gerados servem de apoio ao } \\
\text { planejamento urbano (ATTARD; HAKLAY; CAPINERI, 2016). Em síntese, } \\
\text { crowdsourcing tem a potencialidade de oferecer a governança inteligente, } \\
\text { recolhendo e analisando dados dos cidadãos (HALDER, 2015). }\end{array}$ \\
\hline Geogovernança & $\begin{array}{l}\text { É a governança territorial "esclarecida" (DUBUS; HELLE; MASSON-VINCENT, 2010), } \\
\text { que considera a opinião dos habitantes na construção do planejamento } \\
\text { sustentável (VINCENT, 2008), por meio do conhecimento compartilhado do } \\
\text { território a partir das múltiplas visões de seus atores (sociedade civil, governo, } \\
\text { especialistas) para construir o território, baseado em ferramentas de análise } \\
\text { espacial (MASSON-VINCENT et al., 2011). Portanto, trata-se da aplicação de } \\
\text { métodos e ferramentas de análise espacial participativa e colaborativa que } \\
\text { busca tornar compreensível a complexidade territorial, destacando as questões } \\
\text { socioespaciais, bem como as possíveis evoluções para diferentes horizontes } \\
\text { temporais (DUBUS; HELLE; MASSON-VINCENT, 2010). }\end{array}$ \\
\hline $\begin{array}{l}\text { Governança } \\
\text { Inteligente }\end{array}$ & $\begin{array}{l}\text { Considerada o grande desafio para efetivação das cidades inteligentes (SUJATA } \\
\text { et al., 2016), representa a coleção de tecnologias, pessoas, políticas, práticas, } \\
\text { recursos, normas sociais e informações que interagem para apoiar as atividades } \\
\text { de governo da cidade (CHOURABI et al., 2012), fortemente relacionado à } \\
\text { privacidade, segurança, desempenho econômico, inclusão social e outras } \\
\text { questões assistidas pelas TIC (BATTY et al., 2012), que é a transformadora da } \\
\text { governança em governança inteligente (VIALE PEREIRA et al., 2017b). Inclui as } \\
\text { inovações no governo electrônico e a participação dos cidadãos, aliada a } \\
\text { estruturação econômica da cidade (CARAGLIU; DEL BO; NIJKAMP, 201 1), ou seja, } \\
\text { envolve vários intervenientes na tomada de decisões e serviços públicos (ALBINO; } \\
\text { BERARDI; DANGELICO, 2015) aliado aOS investimentos criativos em tecnologias } \\
\text { emergentes (PRZEYBILOVICZ; CUNHA; TOMOR, 2017). }\end{array}$ \\
\hline Valor público & $\begin{array}{l}\text { É o valor esperado nas ações das organizações públicas (CORTES-MORALES, } \\
\text { 2017). Significa, melhorar a eficiência, eficácia ou a equidade da gestão pública, } \\
\text { introduzindo programas que atendam as aspirações da sociedade ou } \\
\text { reformulando os objetivos da organização para que sejam mais responsivas e } \\
\text { eficazes (MOORE, 1997). Materializado por informações, programas e benefícios } \\
\text { operacionalizados com objetivo de atender as necessidades do público por } \\
\text { meio de governo melhor (HARRISON et al., } 2011 \text { ). Em síntese, criar valor público, } \\
\text { é orquestrar processos de desenvolvimento de políticas públicas, em parceria } \\
\text { com diversos atores, garantindo as boas escolhas segundo interesse público, } \\
\text { legitimando, viabilizando e orientando sua implementação de forma a melhorar } \\
\text { os resultados (BENINGTON; MOORE, 2011). }\end{array}$ \\
\hline
\end{tabular}

Fonte: Autores (2019)

\section{CONCLUSÕES}

Em síntese, constata-se que cidade inteligente requer governança inteligente. Para tanto, as TIC viabilizam a evolução dos governos abertos, tornando-os mais participativos, transparentes, democráticos e responsivos, de modo a permitir além da ativação digital 
(governo eletrônico), a ativação espacial (geogovernança).

Quanto ao uso das inovações disrusptivas, em resposta a questão chave, conclui-se que o uso de crowdsourcing em conjunto aos sistemas de informação geográficas (SIG) efetivam as cidades inteligentes com valor público, visto que possibilitam o exercício do conceito de governo aberto, que compreende aplicar a transparência, a participação e a colaboração em suas ações, acrescidas às práticas de eficiência e eficácia que norteiam a governança urbana inteligente.

\section{AGRADECIMENTOS}

Agradecimento pelo apoio ao desenvolvimento do trabalho à Coordenação de Aperfeiçoamento de Pessoal de Nível Superior - Brasil (CAPES) - Código de Financiamento 001, pelo qual o presente trabalhado foi realizado e, também à COVISA - Coordenadoria de Vigilância em Saúde, do município de Barveri pelo incentivo.

\section{REFERÊNCIAS}

ALBINO, V.; BERARDI, U.; DANGELICO, R. M. Smart cities: definitions, dimensions, performance, and initiatives. Journal of Urban Technology, [s. I.], 2015.

ATTARD, M.; HAKLAY, M.; CAPINERI, C. The potential of volunteered geographic information (VGI) in future transport systems. Urban Planning, [s. I.], v. 1, n. 4, p. 6, 2016.

BASIRI, A.; AMIRIAN, P.; MOONEY, P. Using crowdsourced trajectories for automated OSM data entry approach. Sensors (Switzerland), [s. I.], v. 16, n. 9, p. 1-19, 2016.

BATTY, M. et al. Smart cities of the future. European Physical Journal: Special Topics, [s. I.], v. 214, n. 1, p. 481-518, 2012.

BATTY, M. Big data, smart cities and city planning. Dialogues in Human Geography, [s. I.], v. 3, n. 3, p. 274-279, 2013.

BENINGTON, J.; MOORE, M. H. Public value : theory and practice. [s.I.] : Palgrave MacMillan, 2011.

BOLÍVAR, M. P. R. Characterizing the role of governments in smart cities: a literature review. In: GIL-GARCIA, J. R.; PARDO, T. A.; NAM, T. (Eds.). Smarter as the New Urban Agenda. Cham: Springer International Publishing, 2016. p. 49-71.

BROVELLI, M. A.; MINGHINI, M.; ZAMBONI, G. Public participation in GIS via mobile applications. ISPRS Journal of Photogrammetry and Remote Sensing, [s. I.], v. 114, p. 306-315, 2016.

CALDERON, M.; LOPEZ, G.; MARIN, G. Smartness and technical readiness of Latin American Cities: A critical assessment. IEEE Access, [s. I.], v. 6, p. 56839-56850, 2018.

CARAGLIU, A.; DEL BO, C.; NIJKAMP, P. Smart cities in Europe. Journal of Urban Technology, [s. I.], v. 18, n. 2, p. 65-82, 2011.

CASTELLS, M. A sociedade em rede (a era da informação: economia, sociedade e cultura). São Paulo: Paz e Terra, 2016.

CHOURABI, H.; NAM, T.; WALKER, S.; GIL-GARCIA, J. R.; MELLOULI, S.; NAHON, K.; PARDO, T. A.; SCHOLL, H. J. Understanding Smart Cities: An Integrative Framework. In: 2012 45TH HAWAll INTERNATIONAL CONFERENCE ON SYSTEM SCIENCES 2012, Anais... : IEEE, 2012. 
CORTES-MORALES, R. A comprehensive approach for e-govemment solutions. In: 2017 XLIII LATIN AMERICAN COMPUTER CONFERENCE (CLEI) 2017, Cordoba. Anais... Cordoba: IEEE, 2017. Disponível em: <http://ieeexplore.ieee.org/document/8226452/>

DUBUS, N.; HELLE, C.; MASSON-VINCENT, M. De la gouvernance à la géogouvernance : de nouveaux outils pour une démocratie LOCALE renouveléefrom Governance to GeoGovernance: New Tools for a Renewed Local Democracy. L'Espace Politique, [s. I.], n. 10, 2010. Disponível em: <http://journals.openedition.org/espacepolitique/1574>. Acesso em: 16 mar. 2019.

GIL-GARCIA, J. R.; PARDO, T. A.; NAM, T. What makes a city smart? Identifying core components and proposing an integrative and comprehensive conceptualization. Information Polity, [s. I.], v. 20, p. 61-87, 2015. a.

GIL-GARCIA, J. R.; PARDO, T. A.; NAM, T. A comprehensive view of the 21 st century city: smartness as technologies and innovation in urban contexts. In: GIL-GARCIA, J. R.; PARDO, T. A.; NAM, T. (Eds.). Smarter as the New Urban Agenda. Cham: Springer International Publishing, 2015. b. p. 1-19.

GÓMEZ, E. A. R.; CRIADO, J. I.; GIL-GARCIA, J. R. Public managers' perceptions about open government: a factor analysis of concepts and values. In: PROCEEDINGS OF THE 18TH ANNUAL INTERNATIONAL CONFERENCE ON DIGITAL GOVERNMENT RESEARCH - DG.O '17 2017, Anais... [s.I], 2017.

GOODCHILD, M. F.; GLENNON, J. A. Crowdsourcing geographic information for disaster response: A research frontier. International Journal of Digital Earth, [s. I.], v. 3, n. 3, p. 231-241, 2010.

HALDER, B. Crowdsourcing collection of data for crisis governance in the post-2015 world. In: PROCEEDINGS OF THE 8TH INTERNATIONAL CONFERENCE ON THEORY AND PRACTICE OF ELECTRONIC GOVERNANCE - ICEGOV '14 2014, New York, USA. Anais... New York, USA: ACM Press, 2014. Disponível em: <http://dl.acm.org/citation.cfm? doid=2691 195.2691208>. Acesso em: 30 mar. 2019.

HARRISON, C.; DONNELLY, A. A theory of smart cities. IBM Corporation White Paper, [s. I.], p. 1$15,2011$.

HARRISON, T. M. et al. Open government and e-government: democratic challenges from a public value perspective. In: PROCEEDINGS OF THE 12TH ANNUAL INTERNATIONAL DIGITAL GOVERNMENT RESEARCH CONFERENCE ON DIGITAL GOVERNMENT INNOVATION IN CHALLENGING TIMES - DG.O' 11 2011, New York, USA. Anais... New York, USA: ACM Press, 201 1. Disponível em: <http://dl.acm.org/citation.cfm?doid=2037556.2037597>. Acesso em: 30 mar. 2019.

HEIPKE, C. Crowdsourcing geospatial data. ISPRS Journal of Photogrammetry and Remote Sensing, [s. I.], v. 65, n. 6, p. 550-557, 2010. Disponível em: <http://dx.doi.org/10.1016/j.isprsjprs.2010.06.005>

HERNÁNDEZ-MUÑOZ, J. M. et al. Smart cities at the forefront of the future internet. In: DOMINGUE, J. et al. (Eds.). The Future Internet. FIA 2011. Lecture Notes in Computer Science. LNCS-6656 ed. Berlin, Heidelberg: Springer, 201 1. p. 447-462.

KOMNINOS, N.; PALLOT, M.; SCHAFFERS, H. Developing a Policy Roadmap for Smart Cities and the Future Internet. In: ECHALLENGES E-2011 CONFERENCE PROCEEDINGS 2011, Anais... [s.I]: 2011.

MASSON-VINCENT, M. et al. Information géographique, analyse spatiale et géogouvernance. 
Espace géographique, [s. I.], v. 40, n. 2, p. 127, 2011 . Disponível em:

<https://www.cairn.info/revue-espace-geographique-2011-2-page-127.htm>. Acesso em: 16 mar. 2019.

MCCALL, M. K. Seeking good governance in participatory-GIS: A review of processes and governance dimensions in applying GIS to participatory spatial planning. Habitat

International, [s. I.], v. 27, n. 4, p. 549-573, 2003. Disponível em:

<https://www.sciencedirect.com/science/article/pii/S0197397503000055>. Acesso em: 20 mar. 2019.

MEIJER, A. J.; BOLÍVAR, M. P. R. Governing the smart city: a review of the literature on smart urban governance. International Review of Administrative Sciences, [s. I.], v. 82, n. 2, p. 392408, 2016. Disponível em:

<https://journals.sagepub.com/doi/pdf/10.1177/0020852314564308>. Acesso em: 16 fev. 2019.

MEIJER, A. J.; GIL-GARCIA, J. R.; BOLÍVAR, M. P. R. Smart city research: contextual conditions, governance models, and public value assessment. Social Science Computer Review, [s. I.], v. 34, n. 6, p. 647-656, 2016. Disponível em:

<http://journals.sagepub.com/doi/10.1177/0894439315618890>. Acesso em: 16 mar. 2019.

MOORE, M. H. Creating public value: strategic management in government. [s.I.] : HARVARD UNIVERSITY P, 1997.

NAM, T.; PARDO, T. A. Conceptualizing smart city with dimensions of technology, people, and institutions. In: PROCEEDINGS OF THE 12TH ANNUAL INTERNATIONAL DIGITAL GOVERNMENT RESEARCH CONFERENCE ON DIGITAL GOVERNMENT INNOVATION IN CHALLENGING TIMES DG.O '11 2011, New York, USA. Anais... New York, USA: ACM Press, 2011. Disponível em: <http://dl.acm.org/citation.cfm?doid=2037556.2037602>

OJO, A.; DZHUSUPOVA, Z.; CURRY, E. Exploring the nature of the smart cities research landscape. In: GIL-GARCIA, J. R.; PARDO, T. A.; NAM, T. (Eds.). Smarter as the New Urban Agenda. Cham: Springer International Publishing, 2016. p. 23-47.

PRZEYBILOVICZ, E.; CUNHA, M. A.; TOMOR, Z. Identifying essential organizational characteristics for smart urban governance. In: PROCEEDINGS OF THE 18TH ANNUAL INTERNATIONAL CONFERENCE ON DIGITAL GOVERNMENT RESEARCH - DG.O '17 2017, NeW York, USA. Anais... New York, USA: ACM Press, 2017. Disponível em: <http://dl.acm.org/citation.cfm? doid=3085228.3085281 >

ROCHE, S. Geographic information science I: why does a smart city need to be spatially enabled? Progress in Human Geography, [s. I.], v. 38, n. 5, p. 703-711, 2014. Disponível em: <http://journals.sagepub.com/doi/10.1177/0309132513517365>

RØISELAND, A. Understanding local governance: institutional forms of collaboration. Public Administration, [s. I.], v. 89, n. 3, p. 879-893, 2011. Disponível em: <http://doi.wiley.com/10.1111/j.1467-9299.2010.01887.x>

SANGIAMBUT, S.; SIEBER, R. The $V$ in VGI: citizens or civic data sources. Urban Planning, [s. I.], v. 1, n. 2, p. 141-154, 2016. Disponível em:

<http://www.cogitatiopress.com/ojs/index.php/urbanplanning/article/view/644>

SUJATA, J. et al. Developing smart cities: an integrated framework. In: PROCEDIA COMPUTER SCIENCE 2016, Anais... [s.l: s.n.] Disponível em: <https://linkinghub.elsevier.com/retrieve/pii/S1877050916315022>

THOMPSON, N. K. et al. Democratic innovations in local government: a public value 
perspective. In: SUSTAINABILITY IN PUBLIC WORKS CONFERENCE 2014, Tweed Heads / Coolangatta. Anais... Tweed Heads / Coolangatta

TOWNSEND, A. M. Smart cities : big data, civic hackers, and the quest for a new utopia. 1. ed. [s.l.] : W. W. Norton \& Company, 2013.

VIALE PEREIRA, G. et al. Delivering public value through open government data initiatives in a Smart City context. Information Systems Frontiers, [s. I.], v. 19, n. 2, p. 213-229, 2017. a.

Disponível em: <http://link.springer.com/10.1007/s10796-016-9673-7>

VIALE PEREIRA, G. et al. Increasing collaboration and participation in smart city governance: a cross-case analysis of smart city initiatives. Information Technology for Development, [s. I.], v. 23, n. 3, p. 526-553, 2017. b. Disponível em:

<https://www.tandfonline.com/doi/full/10.1080/02681 102.2017.1353946>

VINCENT, M. Governance and geography explaining the importance of regional planning to in their living. Boletin de la Asociacion de Geografos Espanoles, [s. I.], v. 46, p. 77-95, 2008.

Disponível em: <http://age.ieg.csic.es/boletin/46/05-GOVERNANCE.pdf>. Acesso em: 16 mar. 2019.

VINCENT, M. M. et al. La Géogouvernance : un concept novateur? Cybergeo: European Journal of Geography [En ligne], [s. I.], 2012. Disponível em:

<http://journals.openedition.org/cybergeo/25086> 